\title{
Lo smartworking come opportunità di lavoro per le donne con disabilità fisico-organiche. Esito specifico dell'indagine interna- zionale "Ready Woman. New opportunities of employment for social inclusion of women with disabilities"
}

\author{
Smartworking as job opportunity for women with physical-or= \\ ganic disabilities. Focused results of the international research \\ "Ready Woman. New opportunities of employment for social in- \\ clusion of women with disabilities" \\ Loredana Perla", Laura Sara Agrati ${ }^{* *}$, Alessia Scarinci**, Maria Concetta Rossiello***
}

\begin{abstract}
Riassunto
Oggetto dell'articolo è lo studio delle condizioni lavorative delle donne con disabilità, in particolare delle opportunità offerte dalle tecnologie che consentono di lavorare in remoto. Su quest'ultimo, specifico, tema vengono presentati i risultati di una ricerca sull'accessibilità e su nuovi settori di impiego per le donne con disabilità, in generale, e con disabilità fisica e sensoriale, realizzata nell'ambito del progetto Erasmus+"Ready Woman, New opportunities of employment for social inclusion of women with disabilities" presso il Dipartimento For.Psi.Com. dell'Università degli Sudi di Bari 'Aldo Moro'. L'indagine a metodo misto, condotta con strumenti quali-quantitativi, restituisce le percezioni e le rappresentazioni delle esperienze delle donne con disabilità in cerca di lavoro e degli operatori dei servizi di orientamento/collocazione riguardo le opportunità e le sfide poste dallo smart working.
\end{abstract}

Parole-chiave: telelavoro; donne; disabilità.

\footnotetext{
* Professoressa ordinaria di Didattica e Pedagogia speciale presso 1'Università degli Studi di Bari “Aldo Moro”. E-mail: loredana.perla@uniba.it.

** Professoressa Associata di Didattica e Pedagogia speciale presso l'Università Telamatica "Giustino Fortunato". E-mail: 1s.agrati@unifortunato.eu.

${ }^{* * *}$ Ricercatrice di Didattica e Pedagogia speciale presso l'Università degli Studi di Bari “Aldo Moro". E-mail: alessia.scarinci@uniba.it.

${ }^{* * * *}$ Ricercatrice a t.d. di Didattica e Pedagogia speciale presso l'Università degli Studi di Foggia. E-mail: mariaconcetta.rossiello@unifg.it.

L'articolo è il frutto di un progetto concepito e sviluppato insieme. Tuttavia, nella redazione materiale dei paragrafi il 1 paragrafo è da attribuire a Maria Concetta Rossiello, il 2 ad Alessia Scarinci, il 3 a Laura Sara Agrati, il 4 a Loredana Perla.
} 


\begin{abstract}
The article's topic is the studies of the working conditions of women with disabilities, focusing on the opportunities to remote working, offered by the technologies. Focused results of an investigation on accessibility and new sectors of employment for women with disabilities, in general, and with physical and sensory disabilities - developed within the Erasmus + Project 'Ready Woman, New job opportunities for social inclusion of women with disabilities' by the For.Psi.Com Department at the University of Bari 'Aldo Moro' - are discussed. The mixed-method research, trough quali-quantitative tools, returns perceptions and representations of the women with disabilities looking for work and of the operators of the orientation / placement services regarding opportunities and challenges posed by smart working.
\end{abstract}

Keywords: smartworking; women; disabilities.

\title{
1. Oltre il gender disability gap nel mondo del lavoro: status quo
}

Come è noto, la Convenzione ONU sui diritti delle persone con disabilità, ratificata dal nostro Paese il 24 febbraio del 2009, ha introdotto delle trasformazioni profonde di carattere culturale, sociale, politico e tecnico nel modo di concepire e trattare il tema della disabilità. Trasformazioni di approccio che hanno fatto proprio un modello sociale di disabilità basato sul rispetto dei diritti umani (Perla, 2013; Volpicella, 2017; D’Alonzo, 2018), ben espresso dallo slogan del movimento mondiale delle persone con disabilità "nulla su di noi senza di noi". L'importante novità di tale Convenzione è che, per la prima volta, viene fatta luce sulla discriminazione multipla cui sono soggette le donne con disabilità in ogni angolo del pianeta unitamente alla condizione di invisibilità che spesso le accompagna. L'art. 6, dedicato specificatamente alle donne con disabilità, infatti, così recita: "Gli Stati Parti riconoscono che le donne e le bambine con disabilità sono soggette a discriminazioni multiple e, a questo riguardo, si prenderanno misure per assicurare il pieno ed uguale godimento di tutti i diritti umani e delle libertà fondamentali da parte di donne e bambine con disabilità". E ancora: "gli Stati Parti prendono ogni misura idonea ad assicurare il pieno sviluppo, avanzamento e empowerment delle donne, allo scopo di garantire loro l'esercizio e il godimento dei diritti umani e delle libertà fondamentali enunciate nella presente Convenzione (Testo Unico Convenzione Onu, 2009, p. 9).

A questi indirizzi si aggiunge l'invito, altrettanto significativo, ad incorporare una prospettiva di genere in tutti i progetti a favore delle persone con disa- 
bilità. Essere donne con disabilità non produce, infatti, solo una somma di discriminazioni, quanto una loro moltiplicazione. Esse non godono di pari opportunità né rispetto alle altre donne, né rispetto agli uomini con disabilità. I dati del Gender Gap Global Report (2019) lo confermano. E ancor di più quelli dell'indagine Istat (2019) della quale è utile riportare qualche dato.

Mentre in Europa il 20\% delle donne in età da lavoro risulta disoccupato, in Italia lo è il 32,6\%. L'indagine indica, tra i motivi, la difficoltà nella conciliazione di vita familiare e impegno lavorativo e come modalità risolutiva la preferenza di contratti part-time, flessibilità nell'organizzazione oraria e, specialmente da quanto è stato regolamentato, le modalità in smart working ${ }^{l}$. Ancora meno confortanti sono i dati della popolazione femminile con disabilità: solo il $26,7 \%$ delle donne con disabilità tra i 15 e 64 anni è occupato, rispetto al 36,3\% degli uomini e al $57,8 \%$ del resto della popolazione. Le persone disabili, in generale, sono impiegate nel settore della Pubblica Amministrazione, grazie a programmi di inserimento mirato - L. n. 68/1999 e D.L. n. 81/2015 - e raggiungono livelli mediamente non elevati di carriera $-54 \%$ operai o lavoratori in proprio $(50,4 \%$, resto della popolazione), il $46 \%$ posti di dirigenza o di libera professione $(49,6 \%$, resto della popolazione). Tale dato fa capire che, laddove vi è accesso, diminuisce il gap di possibilità tra disabili e il resto della popolazione e che, pertanto, è necessario un forte intervento in termini di orientamento e formazione. Le statistiche Europee (Eurostat, 2019) confermano che le donne disabili affrontano con più difficoltà l'inclusione socio-lavorativa e professionale in quanto abbandonano più precocemente il sistema di istruzione formale o hanno più difficoltà nell'ottenere qualifiche per l'accesso ai lavori specializzati. Anche per questo, tra gli obiettivi prioritari della policy europee nel settore dell'occupazione vi è quello di offrire alle persone con disabilità e alle donne, in particolare, possibilità di accesso al mercato del lavoro (European Commission, 2017, p. 4) attraverso investimenti sulla qualità della formazione di base e di tipo professionale, nello specifico, sperimentazioni pilota in settori lavorativi emergenti e il ricorso, su scala generale, alla smart economy (Ministero del Lavoro, 2014; Marciano, 2012). L'11 luglio 2018 il Comitato economico e sociale europeo (CESE), l'organo che rappresenta la società civile organizzata dell'UE, ha invitato le istituzioni europee e gli Stati membri a fare

\footnotetext{
${ }^{1}$ Con tale espressione si intende l'insieme delle attività imprenditoriali che ricorrono a reti telematiche, o ITC in generale, per lo svolgimento del lavoro; spesso legate a flessibilità nei servizi forniti e maggiore interconnessione tra dimensione locale e globale - L. n. 81 del 22 maggio 2017.
} 
molto di più per proteggere le donne e le ragazze con disabilità e per accompagnarle efficacemente nella collocazione lavorativa ${ }^{2}$. Come intervenire?

Questi sono alcuni degli obiettivi sui quali il Comitato ha esortato i Paesi membri dell'UE a profondere un impegno mirato:

a) utilizzare i fondi dell'UE per promuovere l'accessibilità e la non discriminazione nei confronti delle donne e delle ragazze con disabilità;

b) applicare il principio della parità di retribuzione a parità di lavoro svolto, a contrastare le discriminazioni salariali e a garantire la parità tra donne e uomini, anche per quanto riguarda le persone con disabilità (pp.4-8).

c) favorire il giusto equilibrio tra vita professionale e vita privata incoraggiando l'accesso all'occupazione come condizione per l'inclusione sociale;

d) definire una legislazione del lavoro specifica che tenga conto delle esigenze delle donne con disabilità in materia di gravidanza e maternità, salvaguardando la permanenza sul mercato del lavoro e garantendo la tutela del lavoro;

e) sostenere e incoraggiare gli Stati membri nell'affrontare la discriminazione generata dall'intersezione tra identità di genere, espressione di genere, orientamento sessuale, caratteristiche sessuali e disabilità, attraverso la formazione alla diversità e la collaborazione con i datori di lavoro per introdurre misure sul posto di lavoro, ad esempio volte a promuovere procedure di assunzione anonime (pp.16-20).

Creare una cultura di lavoro più inclusiva - ma anche condizioni sociali meno ostili all'inserimento lavorativo di donne con disabilità - è, quindi, un presupposto necessario per incamminarsi verso un futuro più equo. Con lo smart working il cammino verso tale direzione può farsi più agevole. Vediamo specificatamente.

\section{L'“e-inclusion" nel mondo del lavoro}

Per favorire l'inclusione lavorativa di adulti con disabilità sarebbe opportuno adottare un approccio "globale" al mondo del lavoro (Perla, 2013). La disabilità, infatti, non è solo una "mancanza" fisica, ma va compresa come la risultanza di una inadeguatezza delle infrastrutture ambientali che non riescono a corrispondere ai bisogni e alle capacità degli utenti, creando così la "situazione di handicap" (Kadouche et al., 2009). I contesti lavorativi non sempre

2 "Le donne con disabilità hanno bisogno di un sostegno ad hoc, eppure non viene loro riservata un'adeguata attenzione in nessuna delle strategie dell'UE, né nella strategia dedicata alle donne né in quella a favore delle persone con disabilità. È come se queste donne non esistessero, come se valessero meno del resto della popolazione", queste le parole del membro del Consiglio del Forum europeo sulla disabilità Gunta Anča dinanzi all'Assemblea plenaria 
risultano idonei, accessibili e/o flessibili, e quindi disposti ad accogliere e favorire l'inserimento del soggetto con disabilità. Come evidenziano Ravicchio, Repetto e Trentin (2015) le aziende, per potersi adeguare alle esigenze e ai bisogni dei suoi lavoratori, devono modificare l'organizzazione del lavoro e adottare "mediatori/facilitatori nella mediazione fra lavoratore disabile e azienda" quali il Disabilty Manager (Ravicchio, Repetto, Trentin, 2015: 103; Mattana, 2016; Fiore, 2018). Questo per accrescere oltre che l'accessibilità al mondo del lavoro, anche la responsabilità sociale delle stesse imprese (Angeloni, 2010; Universal Design; CEN). Il concetto di accessibilità è, poi, strettamente connesso a quello di partecipazione: la società deve poter assicurare a tutti i cittadini, disabili e non disabili, di poter esprimere le proprie potenzialità, di poter esercitare i propri diritti in condizioni di pari opportunità. Secondo Nussbaum la società dovrebbe essere capace di comprendere e sostenere i bisogni dei cittadini affinchè possano svolgere le proprie funzioni in modo attivo e partecipe. Per farlo, tuttavia, essa ha bisogno di riprogettarsi, di eliminare le barriere per poter essere così "autenticamente per tutti" (Colazzo, 2010). L'accessibilità costituisce, secondo il documento della Commissione Europea "e-Inclusion-The Information Society Potential for Social Inclusion in Europe" del 2001, un prerequisito necessario in grado di assicurare a tutte le persone con disabilità i diritti umani e di libertà. La promozione dell'accessibilità lavorativa può essere facilitata dall'implementazione delle tecnologie. La diffusione e la facilità di accesso agli strumenti tecnologici sta modificando i sistemi di comunicazione e di interazione delle persone e tra queste e i diversi contesti di vita, favorendo l'inclusione sociale. Secondo la Convenzione sui Diritti delle Persone con Disabilità (2006) le tecnologie sono un fattore abilitante in quanto promuovono l'autonomia e la partecipazione alla vita sociale, economica e della comunità. Le tecnologie, nel rispetto degli standard e dei principi della progettazione universale (Universal Design), potrebbero contribuire al miglioramento della qualità della vita dei disabili e al loro inserimento nel contesto lavorativo poiché rendono l'attività lavorativa più flessibile e consentono di eliminare le barriere spazio-temporali e i vincoli legati alla mobilità (Ravicchio, Trentin, 2015; Paparella, 2010; Libro Bianco, 2003).

Le possibilità inclusive dei mezzi di comunicazione, come evidenziato nel Background Paper for World Development Report 2016, sono inoltre connesse ad una maggiore disponibilità di "contenuti in più formati e attraverso più media" tra cui le persone con disabilità possono scegliere la modalità di comunicazione più adatta alle proprie capacità e funzionalità (Samant Raja, 2016: 7). Tuttavia non è sufficiente il solo impiego delle tecnologie per colmare le "lacune dell'inclusione socio-economica", ma si rende necessaria una maggiore presa di coscienza e conoscenza delle soluzioni possibili offerte dalle TIC da 
parte dei soggetti interessati e di una progettazione e applicazione degli strumenti che consideri i vincoli richiesti di usabilità e accessibilità (Samant Raja, 2016; Calvani, 2017). Questi vincoli richiedono l'osservazione degli utenti e le loro modalità d'uso dell'oggetto affinchè possa rispondere alle reali esigenze e bisogni espressi nei diversi contesti d'uso e una progettazione che rispetti degli standard definiti che ne conferiscono la conformità (Calvani, 2017; Colazzo, 2010).

\subsection{Le tecnologie assistive}

Come riporta Samant Raja nel "Background Paper" (2016), le soluzioni tecnologiche per le differenti tipologie di disabilità possono essere diverse e comportano lo sviluppo di particolari software e hardware conformi alla misura di accessibilità, come:

\begin{tabular}{|c|c|}
\hline Disabilità & Soluzioni tecnologiche \\
\hline Disabilità visiva & $\begin{array}{l}\text { - } \quad \text { Sintesi vocale } \\
\text { - } \quad \text { Display Braille } \\
\text { - Ingrandimento di schermo e testo (ZoomText e } \\
\text { Magic). } \\
\text { - } \quad \text { Riconoscimento vocale } \\
\text { - } \quad \text { Descrizione audio di supporti grafici e visivi } \\
\text { - Segnaletica audio elettronica } \\
\text { - Navigazione facilitata dal GPS } \\
\text { - Riconoscimento ottico dei caratteri o delle im- } \\
\text { magini } \\
\text { - Modifica della luminosità dello schermo, contra- } \\
\text { sto del colore }\end{array}$ \\
\hline Disabilità uditive & $\begin{array}{l}\text { - Sottotitoli chiusi e aperti, sottotitoli per video, } \\
\text { programmazione TV } \\
\text { - Messaggi di testo } \\
\text { - Telefono di testo o dispositivo di telecomunica- } \\
\text { zione per non udenti (TTY / TDD) che consente l'in- } \\
\text { vio di messaggi di testo tramite la linea telefonica } \\
\text { - Servizi di inoltro delle telecomunicazioni che } \\
\text { consentono al testo di generare conversioni tramite } \\
\text { un operatore } \\
\text { - Utilizzo di vibrazioni / avvisi di testo anziché di } \\
\text { avvisi audio }\end{array}$ \\
\hline Disturbi del linguaggio & $\begin{array}{l}\text { - SMS, messaggi di testo } \\
\text { - Uscita vocale sintetizzata, funzionalità di sintesi } \\
\text { vocale } \\
\text { - Utilizzo di quadro virtuale e soluzioni di comuni- } \\
\text { cazione }\end{array}$ \\
\hline Disabilità fisiche & - Sistemi di riconoscimento vocale \\
\hline
\end{tabular}




\begin{tabular}{|c|c|}
\hline & $\begin{array}{l}\text { - Tastiere adattate e virtuali } \\
\text { - Joystick e mouse adattato } \\
\text { - Uso di occhi e gesti per controllare i dispositivi } \\
\text { - Accesso remoto e online a lavoro, istruzione e } \\
\text { altri servizi }\end{array}$ \\
\hline Disabilità cognitive & $\begin{array}{l}\text { - Rappresentazione da sintesi vocale e output vo- } \\
\text { cale / vocale } \\
\text { - } \quad \text { Dispositivi touch screen } \\
\text { - App mobili e risorse online che imitano i dispo- } \\
\text { sitivi di comunicazione aumentativa e alternativa } \\
\text { (CAA), quadri elettronici per la comunicazione } \\
\text { - Strumenti di organizzazione e supporto della } \\
\text { memoria come calendari online, appunti, avvisi } \\
\text { - Navigazione facilitata dal GPS } \\
\text { - Uso di contenuti multimediali per facilitare la } \\
\text { comprensione, ad esempio video, grafica }\end{array}$ \\
\hline Disabilità psicosociale & $\begin{array}{l}\text { - Utilizzo di comunicazione online, documenta- } \\
\text { zione, strumenti di lavoro per agevolare la pianifica- } \\
\text { zione flessibile } \\
\text { - Strumenti di organizzazione e supporto della } \\
\text { memoria come calendari online, appunti, avvisi }\end{array}$ \\
\hline
\end{tabular}

Nel contesto lavorativo possono essere usate app che aiutano le persone con disabilità uditive attraverso didascalie istantanee per contenuti audio, facilitando videochiamate/webconference; ma ci sono anche servizi forniti dal web che offrono assistenza attraverso sottotitoli live in remoto per riunioni e webinar, interpretazione della lingua dei segni remota e video relay in cui un interprete della lingua dei segni assiste nella comunicazione tra persone con e senza disabilità uditive (Samant Raja, 2016). Come sostiene Paparella (2010), le tecnologie, così intese, possono essere considerate come risorse, facilitatori e mediatori tra le persone con disabilità e il contesto lavorativo, agevolando lo svolgimento dei compiti assegnati ai dipendenti e favorendo un miglioramento dei servizi produttivi. Per facilitare l'inclusione lavorativa "I datori di lavoro pubblici e privati pongono a disposizione del dipendente disabile la strumentazione hardware e software e la tecnologia assistiva adeguata alla specifica disabilità, anche in caso di telelavoro, in relazione alle mansioni effettivamente svolte" (art. 4 comma 4 della L. 4/2004). La presenza e disponibilità di diversi hardware e software consente una personalizzazione della scelta sulla base delle caratteristiche del soggetto disabile, della convenienza dello strumento (funzione e costi) e delle conoscenze pregresse che il soggetto ha rispetto ad esso (AgID, 2015). Le tecnologie assistive da impiegare nei contesti lavorativi sono indicate nella circolare redatta dall'AgID (2015) che riguarda prodotti per la 
comunicazione e l'informazione, per sentire, per parlare, per fare calcoli, software per la comunicazione faccia a faccia, accessori per la telefonia, strumenti di supporto mnemonico, etc.

\subsection{Lo smart work per l'accessibilità}

Il proliferare degli strumenti tecnologici anche nel mondo del lavoro ha portato ad un cambiamento nel modo di comunicare, conoscere e organizzare il lavoro stesso. Dapprima l'uso dei PC, la posta elettronica, internet hanno operato un primo cambiamento ottimizzando le modalità lavorative, semplificando e velocizzando la comunicazione e l'archiviazione dei documenti. Successivamente l'uso degli strumenti informatici, delle telecomunicazioni ha portato allo sviluppo del telelavoro: lavoro in remoto, da casa, in isolamento. Si tratta di una modalità che si basa ancora su modelli centralistici che prevedono poca flessibilità negli orari e negli spazi, come evidenziano Ravicchio, Repetto e Trentin (2015) e il rapporto CISCO (2011), l'uso delle tecnologie consente di gestire uffici satellite (uffici decentrati) controllati da remoto tramite dei software. Per evitare l'isolamento sono poi stati aperti degli spazi di co-working che offrono uno spazio comune, generalmente open space, in cui i teleworker, previo pagamento, possono lavorare e in cui, come scrivono Ravicchio, Repetto e Trentin (2015: 16), "si intrecciano la dimensione fisica, mentale, sociale e virtuale".

Il trasferimento, poi, di pratiche e processi sul cloud, la condivisione di documenti tramite il web o app, maggiore collaborazione nelle attività online, ha portato ad uno spostamento dallo spazio fisico del luogo di lavoro ad uno spazio virtuale che rende più flessibile la programmazione delle attività e rende l'ambiente di lavoro più inclusivo, migliorando l'occupazione delle persone con disabilità (Samant Raja, 2016; Calvani, 2017; Ravicchio, Repetto e Trentin, 2015). Si parla adesso di smart working che in Italia viene definito con la L. n. 81 del 2017 come "modalità di esecuzione del rapporto di lavoro subordinato stabilita mediante accordo tra le parti, anche con forme di organizzazione per fasi, cicli e obiettivi e senza precisi vincoli di orario o di luoghi di lavoro, con il possibile utilizzo di strumenti tecnologici per lo svolgimento dell'attività lavorativa. La prestazione lavorativa viene eseguita, in parte all'interno di locali aziendali e in parte all'esterno senza una postazione fissa, entro i soli limiti di durata massima dell'orario di lavoro giornaliero e settimanale, derivanti dalla legge e dalla contrattazione collettiva". In questa nuova modalità di lavoro la rete e le tecnologie costituiscono l'elemento principale che facilita e ottimizza il lavoro, eliminano le barriere legate allo spazio e al tempo rendendo il rapporto di lavoro più flessibile e agevole: "[...] consente alle persone di lavorare in 
modo più intelligente, ma influenza e determina anche i loro atteggiamenti personali nei confronti del lavoro: dove e quando lavorano, e come competono, collaborano, comunicano per scambiare informazioni e prevedere nuovi approcci al lavoro e ai processi di innovazione" (CISCO, 2011:3).

Le tecnologie consentono la partecipazione alla cultura del lavoro, un maggiore scambio all'interno dell'organizzazione, e sono promosse forme di collaborazione e condivisione del know how.

Ravicchio, Repetto e Trentin (2015) sulla base di quanto emerso da vari studi, hanno individuato le tre dimensioni dello smart work: dimensione del tempo, dimensione dello spazio e dimensione della tecnologia.

- Dimensione spaziale: le tecnologie hanno portato ad un graduale sgretolamento dei confini dei luoghi fisici del sociale e del lavoro, si estendono nello spazio che diventa sempre più virtuale, uno spazio aperto e condiviso in cui produrre conoscenza e svolgere il proprio lavoro in modo indipendente rispetto alla posizione in cui ci si trova e al dispositivo impiegato (CISCO, 2011)

- Dimensione temporale: la perdita di un luogo fisico in cui svolgere il lavoro comporta una maggiore flessibilità anche dei tempi del lavoro, anche se le definizioni in rapporto alla questione tempo sono diverse e molteplici (soglia minima di ore a distanza, parte o intero orario da svolgere a distanza,...), l'aspetto innovativo è legato in particolar modo al rapporto tempo e obiettivi da raggiungere, ovvero "il tempo non è più concepito come una unità di misura per determinare l'impegno del lavoratore nella sua mansione. Al contrario, diventa un riferimento per valutare l'efficienza dei processi e un elemento centrale per la distribuzione di mansioni" (Ravicchio, Repetto, Trentin, 2015: 18).

- Dimensione tecnologica: quest'ultima dimensione è quella che ha reso possibile il cambiamento nelle due precedenti dimensioni. È considerata come sviluppo tecnologico che ha portato all'evoluzioni di modalità di lavoro innovative e a distanza grazie alle potenzialità crescenti offerte dagli strumenti tecnologici.

All'esplorazione dell'uso e delle percezioni connesse allo smart working da parte delle donne con disabilità organico-sensoriali è stata dedicata una specifica parte del progetto di ricerca 'Ready Women - New opportunities of employment for social inclusion of women with disabilities' finanziato con fondi del Programma Erasmus+, Key Action - Cooperation for innovation and the exchange of good practices, Action - KA204, Strategic Partnerships for adult education, promosso dall'associazione non profit FAMS-COCEMFE di Siviglia della federazione provinciale delle Associazioni delle persone con disabilità fisica e organica e che ha coinvolto nove partner fra i quali tre Università col coordinamento scientifico della prof.ssa Loredana Perla (Università degli 
Studi di Bari Aldo Moro, Università 'Gelisim' di Istanbul, Università Telematica 'Giustino Fortunato' di Benevento). Il progetto 'Ready Woman' è nato per offrire alle donne con disabilità - in particolare organica e fisica - nuove opportunità di impiego lavorativo e di sviluppo delle proprie capacità allo scopo di migliorarne i livelli di occupabilità. È stato avviato nell'ottobre 2018, per una durata di 30 mesi ed è, allo stato attuale, al termine della II fase. Le azioni dei nove partner provenienti da sette Paesi $^{3}$ mirano a costruire un modello di inclusione sociale e lavorativa per donne con disabilità, attraverso la sperimentazione di pratiche di orientamento/supporto nell'accesso ai settori di impiego emergenti (definiti con l'acronimo New Yields of Employment - NYE) e di un curricolo inclusivo di formazione mirata sulle competenze trasversali e funzionali di tipo tecnologico e informatico (da sviluppare nella seconda parte della ricerca). Uno degli obiettivi prioritari del progetto è stato quello di individuare le relazioni esistenti tra le condizioni di disabilità e le opportunità di lavoro nei nuovi settori emergenti, in particolar modo riguardo il settore del telelavoro e/o dello smart working, e le risultanze di questa prima fase vengono qui di seguito presentate.

${ }^{3}$ I nove partner rappresentanti di sette Paesi europei sono:

- FAMS-COCEMFE (Federazione Provinciale delle Associazioni delle Persone con Disabilità Fisica e Organica di Siviglia e Confederazione Spagnola delle Persone con Disabilità Fisica e Organica - capofila del Progetto).

- EFE (Ecological Future Education, Lituania): Organizzazione Non Profit che si occupa di formazione attraverso uno stile di vita ecologico, per persone con disabilità e per giovani, adulti e anziani a rischio di esclusione sociale.

- EYES (Education and Youth Empowerment Society - Bulgaria) organizzazione non profit che si occupa di orientamento e inserimento lavorativo dei giovani, formazione all'imprenditorialità, cittadinanza attiva e prevenzione del disagio sociale.

- ERFC (Ruropean Regional Framework for Co-operation - Grecia), Organizzazione Non Profit che si occupa di innovazione e sviluppo economico (sistemi produttivi locali, miglioramento delle risorse territoriali).Eurokom (Italia), organizzazione non profit che si occupa di sviluppo locale e promozione della politica comunitaria attraverso servizi di comunicazione, consulenza fiscale, elaborazione dei progetti.

- 'Gelisim' Università di Istanbul, Dipartimento di Economia, Project maanging.

- Istituto dei Sordi di Torino (Italia), storica fondazione che si occupa, tra l'altro, di programmi di formazione e orientamento professionale per persone con problemi di udito, problemi linguistici e cognitivi.

- Previform (Laboratório, Formação, Higiene e Segurança no Trabalho - Portogallo): azienda con esperienza nel campo della formazione degli adulti, sicurezza sul lavoro, sicurezza alimentare e igiene e nutrizione.

- SEADDER (Surekli Egitim, Arastirma ve Danisma Dernegi - Turchia) associazione senza fini di lucro che si occupa di formazione per adulti, servizi di supporto psicologico ed educatori per adulti, formazione professionale nell'area delle ICT. 


\section{Lo smart work nelle rappresentazioni delle donne disabili e delle operatrici dei servizi}

Vengono descritti di seguito l'impianto metodologico, le procedure di analisi nonché gli esiti dello studio diagnostico ed esplorativo rispettivamente della I e della II fase del Progetto focalizzati sulle percezioni e le esperienze delle donne con disabilità fisica e organica e gli operatori del settore ${ }^{4}$ riguardo opportunità ma anche sfide poste dal telelavoro e dallo smart working.

È stato effettuato uno studio diagnostico più generale che, attraverso la relazione tra le percezioni e le esperienze delle donne con disabilità fisico/organica e le opportunità di lavoro nei settori lavorativi emergenti in ciascuno dei Paesi europei partner nel progetto ottenesse come risultati:

a. una ricognizione dei settori lavorativi maggiormente favorevoli di impiego delle donne con disabilità fisica e organica - attraverso l'analisi della letteratura e dei documenti ufficiali e la somministrazione di un questionario 'ad hoc' rivolto a donne con disabilità in cerca di lavoro e a operatori/responsabili di centri/agenzie per l'impiego;

b. un'analisi dei bisogni formativi che le donne con disabilità fisico/organica esprimono rispetto a specifici settori di impiego - attraverso un'indagine specifica sulle percezioni e le esperienze riguardo i supporti e le barriere nell'accesso al lavoro funzionale all'individuazione di possibili competenze-target verso le quali dirigere l'intervento formativo da realizzare nella seconda fase del progetto.

La trattazione delle procedure di indagine, di analisi e degli esiti di seguito riportate si focalizzano sulle modalità di lavoro in smart working.

\section{Il disegno di indagine}

Nella prima fase del Progetto 'Ready Woman' è stata effettuata un'indagine diagnostica con disegno a metodo misto (Creswell et al., 2003; Leech \& Onwuegbuzie, 2008; Cameron, 2015) con lo scopo di individuare costanze e categorie emergenti in riferimento all'oggetto di studio (supporti e barriere nell'accesso al lavoro) e al telelavoro/smart work, nello specifico. Nello specifico, il disegno (Fig. 1) ha seguito un impianto sequenziale con prima fase 'esplorativa' (Creswell et al., 2003) di raccolta dei dati qualitativi (tramite focus group), seconda fase 'incorporata' di raccolta dei dati quanti- (tramite questionario) qualitativo (tramite interviste in profondità) e un'ultima fase di sintesi delle meta-inferenze, ancora in corso (Cameron, 2015).

\footnotetext{
${ }^{4} \mathrm{Si}$ intendono i consulenti e gli esperti con funzione di orientamento al lavoro, che prestano servizio come dipendenti, autonomi o volontari presso i centri per l'impiego, le associazioni specializzate e le agenzie pubbliche o private.
} 
Tabella 2 - Progetto 'Ready Woman': disegno sequenziale a metodo misto

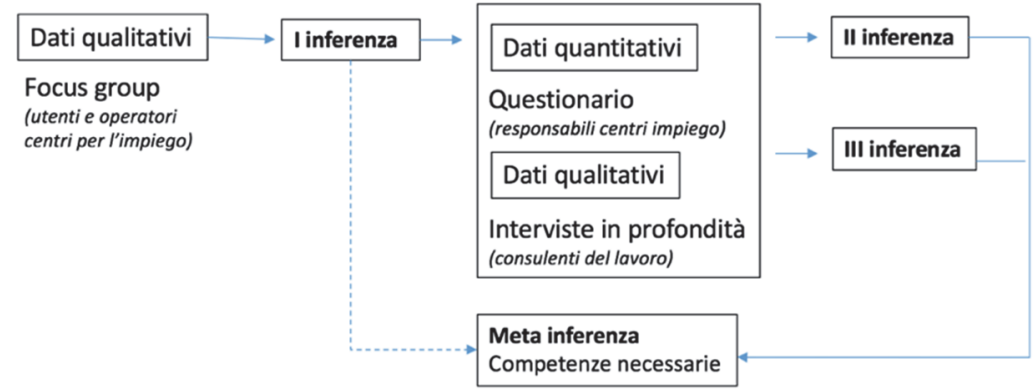

L'impiego lavorativo per le donne con disabilità si presenta come un fenomeno complesso, multifattoriale, con un ventaglio di aspetti che vanno dalle emozioni e le aspettative delle donne coinvolte, alle condizioni dell'ambiente fisico-materiale e socio-culturale di riferimento - es. atteggiamenti, istituzioni, norme sociali e leggi (Sellevoll, 2016). Il rapporto disabilità e impiego lavorativo è oggetto di indagine complesso (Sallis \& Birkin, 2014) di numerose discipline, le quali offrono spunti e direzioni di intervento alle scienze dell'educazione e alla formazione degli adulti, nello specifico (Gottlieb, Myhill, Blanc, 2010; Cairo et al., 2010; Cairo e Marrone, 2017; Vornholt et al., 2017; Cottini et al., 2016). I diversi modelli descrittivi della disabilità e dei processi lavorativi hanno prodotto nel tempo concetti e i costrutti teorici di base numerosi e non sempre conciliabili dal punto di vista delle aree semantiche rappresentate (Vedeler, 2014; Sellevoll, 2016). Il modello bio-psico-sociale del funzionamento (OMS, 2001; 2017) distingue, come noto, tra menomazioni - legate a funzioni e strutture corporee -, limitazioni - legate all'esecuzione di un'attività -, nonché facilitatore/barriera-ostacolo - ossia le condizioni dell'ambientale fisico o sociale, ampiamente intese, tra le quali gli atteggiamenti personali, i servizi offerti dal territorio, da non confondere tuttavia con i 'fattori personali' 5 . Il modello delle capacità (Sen, 1999; Mitra, 2006; Trani et al., 2011; Robertson, 2018), invece, presenta un approccio più ampio alle difficoltà/opportunità di lavoro nella disabilità individuando significati desumibili dalle esperienze e dalle percezioni di quanti vengono coinvolti ${ }^{6}$.

${ }^{5}$ L'International Classification of functioning definisce tra i 'fattori personali' il sesso, la razza, l'età, come anche lo stile di vita, le abitudini, l'educazione ricevuta - intesa come istruzione di base e formazione professione - nonché il background sociale, e l'esperienza di vita, in generale (OMS, 2007, pp. 148-9).

${ }^{6}$ Robertson (2018) mette in evidenza che, dal punto di vista della persona disabile, assume medesimo peso tanto la mancanza di qualifiche e competenze, quanto l'immagine di sé come persona non capace di eseguire un compito spesso, legata all'immagine corporea, 
Lo studio diagnostico-esplorativo della prima fase del Progetto ha inteso far emergere tanto i fattori 'ambientali' quanto quelli 'personali' tramite ampia notazione semantica che definisse l'oggetto di indagine-opportunità e difficoltà ${ }^{7}$ di accesso all'impiego lavorativo. Queste, infatti, possono essere ricavate sulla base di indagini statistiche e dai costrutti offerti dalla ricerca specialistica e operativizzate tramite strumenti di rilevazione quantitativa (es. questionario), oppure desunte a posteriori in ragione dei significati personali e dei contesti di vita emergenti dalle narrazioni (Atkinson, 1998; Jedlowski, 2000) e recepite per via di strumenti come le interviste in profondità o i focus group (Brinkmann \& Kvale, 2015; Sellevol, 2016; Robertson, 2018).

L'impatto della così detta 'tecnologia intelligente' sulla qualità della vita $\mathrm{e}$ l'indipendenza delle persone, in generale, e, nello specifico, di anziani e disabili è ormai noto (Mann, 2005); meno noti, invece, gli effetti sul segmento specifico delle donne con disabilità - cfr. le esperienze di indagine di organizzazioni nonprofit come il britannico Business Disability Forum (Adenipekin, 2020).

Presentiamo di seguito i tre strumenti di rilevazione dei dati quantitativi e qualitativi, le rispettive analisi (Fig. 1) e le evidenze parziali in merito alle opportunità e alle difficoltà nell'impiego in smart working che le donne con disabilità e gli operatori dei servizi hanno restituito.

\section{Gli strumenti di indagine e analisi dei dati}

Come chiarito nel disegno della ricerca (Fig. 1), l'indagine a metodo misto è stata condotta con strumenti quali-quantitativi e restituisce le rappresentazioni delle donne con disabilità in cerca di lavoro (focus group) e delle operatrici dei servizi di orientamento/collocazione (interviste in profondità e questionari) riguardo le opportunità ma anche le sfide poste dal telelavoro e smart working.

Come noto, il focus group consente la condivisione della propria conoscenza/esperienza da parte dei partecipanti attraverso una discussione guidata (Frisina, 2010). Nella prima fase dello studio realizzato per il progetto 'Ready Woman', il focus group ha permesso di accedere ad aspetti personali relativi alle opportunità/difficoltà di inserimento nel lavoro da parte delle donne con disabilità, ulteriori rispetto a quanto evidenziato nel framework della letteratura. Le

alla mancanza di fiducia in sé, al peso delle responsabilità nei confronti di parenti anziani o socialmente in posizione di autorità. Adams (Adams et al., 2018), invece, evidenzia che, dal punto di vista di chi coordina i programmi di inserimento conta maggiormente le disponibilità e la flessibilità nell'organizzazione lavorativa, oltre che le competenze di base.

${ }^{7}$ Lo studio su dati statistici condotto da Coleman, Sykes e Groom (2013) ricorre al temine ampio 'limitazioni' in riferimento alle difficoltà derivanti da: condizioni di salute e psicologiche - come l'ansia e la mancanza di fiducia; atteggiamenti non favorevoli dei datori di lavoro e dei colleghi; barriere architettoniche e la mancanza di tecnologie di supporto); responsabilità rispetto al ruolo familiare. 
sessioni di focus group hanno coinvolto in tutto 106 donne con un'età media di 45,2 anni e un livello di istruzione alto - cfr. Tab. 2.

\begin{tabular}{|c|c|c|c|c|}
\hline Partner & n. partecipanti & n. inoccupate 8 & $\begin{array}{l}\text { livello di } \\
\text { istruzione }^{9}\end{array}$ & $\begin{array}{l}\text { media } \\
\text { età }\end{array}$ \\
\hline SEADDER (Turchia) & 19 & n.p. & Terziaria (14) & 32,4 \\
\hline FAMS-COCEMFE (Spagna) & 17 & 10 & $\begin{array}{l}\text { Secondaria } \\
\text { (9) }\end{array}$ & 45 \\
\hline EYES (Bulgaria) & 17 & 9 & Terziaria (10) & 52,3 \\
\hline ERFC (Grecia) & 14 & 9 & Terziaria (10) & 41,8 \\
\hline EFE (Lituania) & 12 & 3 & $\begin{array}{l}\text { Secondaria } \\
\text { (8) }\end{array}$ & 59 \\
\hline PREVIFORM (Portogallo) & 10 & 6 & $\begin{array}{l}\text { Secondaria } \\
\text { (5) }\end{array}$ & 49,6 \\
\hline EUROKOM & 11 & 6 & Terziaria (8) & 36,9 \\
\hline IST (Italia) & 6 & $1^{*}$ & Terziaria (4) & 36 \\
\hline Totale & 106 & 44 & Terziaria & 45,2 \\
\hline
\end{tabular}

Il materiale testuale reso dalle trascrizioni delle sessioni di focus group è stato tradotto dalle rispettive lingue originali e sottoposto ad una prima sintesi tramite format condiviso che mettesse in evidenza i temi, tra i quali le rappresentazioni sul telelavoro, e una prima analisi di tipo-SWOT (Horn-Haacke, 2002) con punti di forza e debolezza.

L'intero corpus testuale è stato successivamente analizzato con software Nvivo (Lewins \& Silver 2007; Pacifico \& Coppola 2010; Bazekey \& Jackson 2013) e tramite procedura Qualitative Data Analisis (QDA) (Strauss \& Corbin, 1990; Charmaz, 2005; Tarozzi, 2008) scandita nelle tre fasi di open coding (prima concettualizzazione in unità di testo significative ed etichette), axial coding (individuazione di macrocategorie frequenti) e selective coding (gerarchizzazione delle macrocategorie ed emersione delle core category). Riportiamo di seguito le axial coding differenziate per partner.

Come si mette in evidenza nella tabella, le rappresentazioni delle donne con disabilità in cerca di lavoro non pongono una sostanziale differenza tra telelavoro e smart working e, in particolare, ne offrono una fotografia che potremmo definire 'in bianco e nero'.

${ }^{8}$ Stato di non occupazione di oltre 2 anni.

${ }^{9}$ Livello di istruzione maggiormente rappresentato; riferimento all'International Standard Classification of Education (ISCED) - UNESCO-UIS, 2011 che indica come 'istruzione terziaria' quella riferita alla laurea breve, di I e II livello (Master) nonché il dottorato di ricerca. 
Education Sciences \& Society, 1/2020 ISSN 2038-9442, ISSNe 2284-015X

Tabella 4 - Axial coding: macrocategorie differenziate per partner

\begin{tabular}{|c|c|c|}
\hline & Axial coding & \\
\hline Partner & Opportunità & Difficoltà \\
\hline SEADDER (Turchia) & Pro (opportunità di connessione). & $\begin{array}{l}\text { Contro (isolamento, necessità di } \\
\text { infrastrutture). }\end{array}$ \\
\hline FAMS-COCEMFE (Spagna) & $\begin{array}{l}\text { Offre opportunità nel campo della } \\
\text { contabilità, del web design, della } \\
\text { grafica, delle traduzioni. }\end{array}$ & $\begin{array}{l}\text { Non è chiaro di cosa si tratti. } \\
\text { Sono necessarie competenze ITC } \\
\text { specifiche e regolazioni normative } \\
\text { per garantirne l'efficacia. }\end{array}$ \\
\hline EYES (Bulgaria) & $\begin{array}{l}\text { Si sta diffondendo soprattutto tra } \\
\text { le piccole imprese, in particolare } \\
\text { nei servizi (legale, redazione e } \\
\text { traduzione, contabilità). } \\
\text { Richiede un tipo di lavoratore più } \\
\text { affidabile di quello presente (per- } \\
\text { ché è meno "controllabile") che } \\
\text { può essere pagato alla fine } \\
\text { dell'attività e non per ore. }\end{array}$ & $\begin{array}{l}\text { Non è chiaro cosa sia. } \\
\text { Spesso non hanno protezioni so- } \\
\text { ciali e sindacali (non ci sono dati } \\
\text { statistici) e si finisce per andare } \\
\text { nella clandestinità. }\end{array}$ \\
\hline ERFC (Grecia) & II telelavoro è un'opportunità & $\begin{array}{l}\text { II telelavoro è un rischio (isola- } \\
\text { mento del lavoratore). } \\
\text { I datori di lavoro non sono a favore } \\
\text { del telelavoro. } \\
\text { Presenta problemi di accessibilità } \\
\text { e scarse risorse economiche. }\end{array}$ \\
\hline EFE (Lituania) & II telelavoro è utile & $\begin{array}{l}\text { Necessita di competenze e infor- } \\
\text { mazioni specifiche sulle opportu- } \\
\text { nità. } \\
\text { A volte è confuso con il telemarke- } \\
\text { ting e quindi denigrato. }\end{array}$ \\
\hline PREVIFORM (Portogallo) & $\begin{array}{l}\text { II telelavoro è positivo. Facilita } \\
\text { l'accesso al mercato del lavoro. }\end{array}$ & $\begin{array}{l}\text { II telelavoro è negativo. } \\
\text { Limita la partecipazione alle rou- } \\
\text { tine lavorative. } \\
\text { Per diffonderlo, è necessario inve- } \\
\text { stire in aziende (in particolare la } \\
\text { disponibilità dei datori di lavoro) e } \\
\text { l'educazione di competenze spe- } \\
\text { cifiche. }\end{array}$ \\
\hline EUROKOM (Italia) & $\begin{array}{l}\text { È un'opportunità formativa per le } \\
\text { donne con disabilità fisiche che } \\
\text { vivono in villaggi remoti. }\end{array}$ & $\begin{array}{l}\text { II telelavoro non è molto diffuso a } \\
\text { causa delle scarse infrastrutture } \\
\text { tecnologiche (cfr. banda larga). }\end{array}$ \\
\hline IST (Italia) & Ė definito come un vantaggio. & $\begin{array}{l}\text { Non c'è familiarità con il telela- } \\
\text { voro. } \\
\text { Legato a condizioni di necessità } \\
\text { (es. lavorare da casa a causa di } \\
\text { un incidente). } \\
\text { Legato a condizioni specifiche. } \\
\text { Espone al rischio di isolamento } \\
\text { personale e sociale. }\end{array}$ \\
\hline
\end{tabular}


Le opportunità offerte dal telelavoro sono descritte in termini alquanto generici (cfr. SEADDER, ERFC, EFE, IST) e, in alcuni casi, non sono ben note le caratteristiche (cfr. FAMS, EYES), tanto da essere confuso con il telemarketing (cfr. EFE). Solo in due casi il telelavoro viene associato a specifici settori di impiego come la grafica, la contabilità e la traduzione (cfr. FAMS, EYES).

I riferimenti ai rischi, invece, connessi al telelavoro sono abbastanza ricorrenti: una generale condizione di isolamento personale e sociale (cfr. SEADDER, ERFC, IST) che negherebbe, in particolare, i vantaggi della partecipazione alle routines lavorative (cfr. PREVIFORM); questioni di ordine contrattuale e retributivo (cfr. lavoro clandestino, EYES) data l'incerta regolamentazione in merito.

Altri due limiti associati al telelavoro sono la non disponibilità delle infrastrutture - mezzi tecnologici e problemi di connettività (cfr. SEADDER, EUROKOM) - e l'atteggiamento ostativo dei datori di lavoro (cfr. ERFC, PREVIFORM).

Rispetto ai Paesi si evidenzia una considerevole eterogeneità legata soprattutto alla media dell'età e al background educativo. Notiamo una sostanziale differenza tra le donne anziane e le giovani soprattutto riguardo le abilità tecnologiche necessarie per ricorrere al telelavoro (nel primo caso percepite come un limite, nel secondo come un'opportunità - es. cfr. PREVIFORM e EFE, da un lato e EUROKOM, dall'altra), nonché tra le donne con un grado di istruzione secondario e post-secondario riguardo soprattutto le ricadute - se non proprio, i rischi - in termini contrattuali e retributivi del telelavoro - es. cfr. EFE e EYES).

Il contesto territoriale di residenza è un ulteriore aspetto che sembra incidere sulle percezioni circa le opportunità offerte dalle risorse online, in generale, e dal telelavoro, nello specifico: le donne che vivono in contesti urbani sembrano meno portate a cogliere l'isolamento, quale principale inconveniente del telelavoro, a differenza delle abitanti nei territori di campagna (es. cfr. EUROKOM e IST).

Le rappresentazioni emerse tramite focus group sul telelavoro sono, di fatto, contrastanti: da un lato vengono descritte le opportunità - in termini di accesso al mercato, di investimenti circa le competenze tecnologiche ecc. -, dall'altro, vengono richiamati i limiti alla sua attuazione - inadeguato livello normativo e delle infrastrutture, non volontà dei datori di lavoro - e ad esso connessi - il rischio di isolamento sociale.

Il telelavoro è percepito come opportunità ai fini di un collocamento lavorativo - "uno strumento di lavoro utile per le donne con disabilità, perché combina l'accessibilità con il risparmio di denaro, dato che le donne lavorano comodamente da casa" (ERFC, n. 15) - ma anche come limite in quanto "finisce per limitare ancora di più le persone con disabilità, perché rende impossibile 
vivere una routine (soprattutto del lavoro) uguale a quella del resto della società" (PREVIFORM, n. 47).

Tale quadro sembra suggerire un intervento a due livelli rispetto agli agenti del fenomeno - donne con disabilità ma anche datori di lavoro: il primo, di informazione generale sul telelavoro e sulle opportunità connesse - che aiuti a superare le esitazioni e il pregiudizio espresso a livello personale di giudizio ; il secondo, di successivo investimento riguardo le competenze strumentali di accesso (tecniche-tecnologiche) e professionali di servizio (legate a settori lavorativi specifici, es. l'utilizzo di software gestionale).

Va rilevato che l'assenza di necessarie competenze nell'utilizzo strumentale delle tecnologie, implicate nell'accesso al telelavoro è avvertita a volte come limite - "che le competenze informatiche sono uno dei maggiori problemi per le persone con disabilità" (EFE, n. 1) - da superare attraverso intanto un'adeguata informazione circa le potenzialità offerte in modo superare le diffidenze provate dalle stesse donne con disabilità - condizionate rispetto al 'senso di isolamento' - e dai datori di lavoro che "non sono facilmente aperti a questo tipo di lavoro" (ERFC, n. 19, PREVIFORM, n. 47) il cui allestimento è avvertito come costoso.

Prevale, pertanto la posizione ambivalente nei confronti del telelavoro, in generale, e della tecnologia, nello specifico, come ben espresso nel seguente passaggio: "se (la tecnologia) da un lato ha distrutto le barriere esistenti, dall'altro ne ha create di nuove che non esistevano. Se da un lato facilita, dall'altro ostacola. Nel complesso, la tecnologia si è evoluta per rendere la vita delle persone con disabilità più facile e più piacevole, trasformando compiti piuttosto difficili in compiti molto più semplici, trasformando le loro disabilità in azioni" (PREVIFORM, n. 46).

Come noto, scopo di un'intervista è di accedere al mondo della vita, all'esperienza vissuta (Creswell, 2014) di persone scelte proprio in base alla rappresentatività 'sostantiva' ${ }^{10}$ che deterrebbero rispetto all'oggetto di indagine. Nelle indagini sulle esperienze lavorative nei casi di disabilità (Brinkmann \& Kvale 2015; Bryman, 2012;) non è infrequente estendere lo studio anche alle rappresentazioni dei diversi attori che impattano sul fenomeno, come gli operatori del settore, i datori di lavoro, i familiari ecc.

Nella seconda fase dell'indagine del Progetto (cfr. Fig. 1), sono state indagate tramite interviste in profondità le percezioni e gli atteggiamenti di 22 operatori del settore ${ }^{11}$ riguardo specifici aspetti nell'accesso al mercato del lavoro

${ }^{10}$ Per la ricerca sociale (Gobo, 2004; Corbetta, 2003), si tratta della rappresentatività che si ottiene in base a parametri stabiliti dal ricercatore e rispondenti al profilo sociologico studiato.

${ }^{11}$ Consulenti ed esperti nel supporto alla ricerca di lavoro, in servizio presso agenzie pubbliche o private, associazioni specializzate o centri per l'impiego. 
da parte delle donne con disabilità. Le intervistate - 19 donne e 3 uomini svolgono attività in prevalenza autonoma o volontaria (n. 19; n. 3 sono dipendenti) per conto di associazioni di categoria o centri per l'impiego.

Il materiale testuale è stato analizzato tramite software Nvivo dal quale si è potuto ricavare categorie descrittive (Pacifico \& Coppola 2010) sotto forma di 'unità significative' (sources), relazioni tra codici (relationships) e reti di collegamento tra codici (tree nodes).

Il nodo delle opportunità offerte dall'impiego attraverso le nuove tecnologie ricorre 11 volte in 11 interviste (su un totale di 14); in questo sono stati fatti rientrare i riferimenti diretti e indiretti al telelavoro e allo smart working come il seguente:

' $i$ settori che per la mia esperienza offrono maggiori probabilità di impiego per donne con problemi (...) sono il settore amministrativo e quello dei servizi (...) il settore produttivo (...) o ancora lavorare da casa, via Internet, o in attività di lavoro autonomo'

Il nodo riguardante i settori attualmente favorevoli l'impiego delle donne con disabilità ricorre 16 volte in 13 interviste (su un totale di 14) con il riferimento allo smart working associato a compiti di segreteria, operatore telefonico o dell'amministrazione: 'Lavoro autonomo (vendita via Internet; artigianato e artigianato) (...) Le donne con disabilità visive lavorano principalmente nei call center'; 'Lavoro individuale, sul posto di lavoro agevolato a casa attraverso l'intermediazione dell'Agenzia statale; Tutti i campi'; 'Lavoro di segreteria; operatore telefonico; Amministrazione e lavoro di segreteria; segreteria; Tecnico amministrativo'.

Gli operatori intervistati ritengono che le opportunità di lavoro più favorevoli per le donne con disabilità siano quelle, in generale, che richiedono l'uso di strumenti tecnologici e nuove tecnologie, soprattutto nel settore del business, dell'amministrazione e dei servizi e che sono direttamente legate a modalità di telelavoro o in smart working.

Tale modalità, invece, è associata a specifici settori come i servizi pubblici - specie nei Paesi in cui è attiva un'agenzia nazionale per l'occupazione - nonché le vendite online anche private.

Gli operatori si dimostrano generalmente ottimisti rispetto alle opportunità, tanto da affermare che: 'Non ci sono limitazioni di alcun tipo riguardo le opportunità di lavoro se si valutano le possibilità di ogni persona e non i limiti'. Essi considerano che le tecnologie davvero favoriscono le donne con disabilità nel superamento dei limiti fisici (es. le distanze), delle distanze nonché delle problematiche logistiche (es. conciliazione vita-lavoro); per questo si dichiarano molto favorevoli nell'investire in termini di competenze strumentali di accesso e professionali di servizio. Tale atteggiamento in definitiva 'positivo' trova riscontro nel fatto che anche quando gli operatori vengono chiamati ad 
esprimersi riguardo settori lavorativi potenzialmente favorevoli, il più delle volte non ne indicano di specifici - 'tutti $i$ campi' - oppure rimandano a un ventaglio molto ampio.

Lo studio ha effettuato anche un'indagine sulle percezioni degli operatori dei servizi tramite questionario 'ad hoc'12 somministrato on-line. Sono stati coinvolti n. 36 operatori con un'anzianità nella consulenza lavorativa per donne con disabilità fisico-sensoriale in media di 5-10 anni (50\%), che lavora in agenzie/servizi pubblici (50\%) con contratti da dipendente (94\%).

Come fattori maggiormente influenti sull'occupazione per le donne con disabilità fisiche/sensoriali, gli operatori considerano (cfr. domanda n. 20 - a risposta chiusa con più alternative di risposta) principalmente la possibilità di accedere a nuove forme di lavoro - in primo luogo lo smart work e telelavoro - n. 54 occorrenze totali -, prima ancora che il livello di istruzione.

\section{Tabella 5 - Domanda n. 20 - Fattori che incidono sull'occupazione}

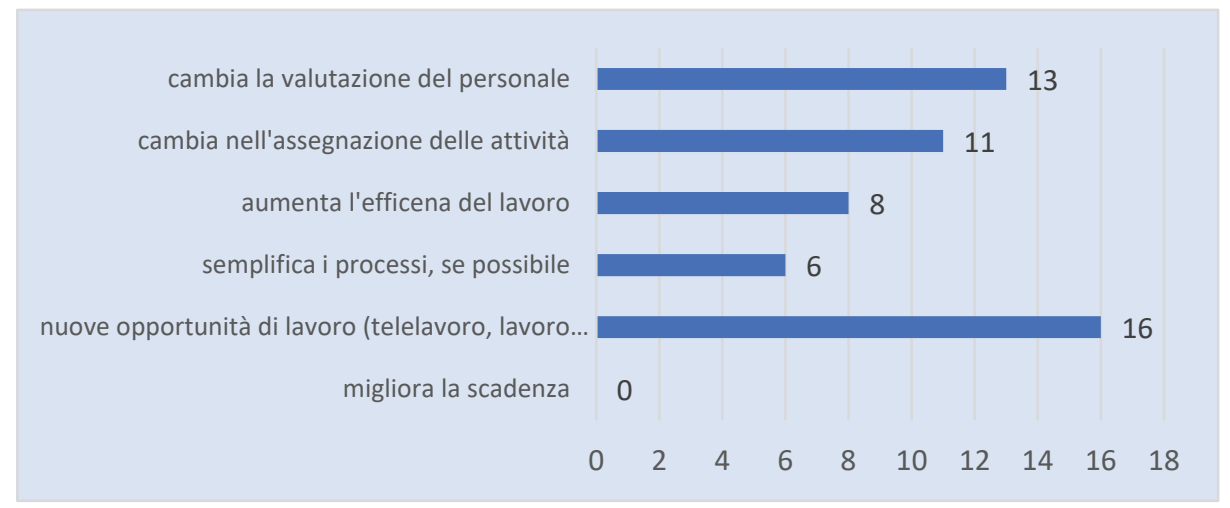

Ritengono, di converso, che proprio la mancanza di modalità di lavoro flessibile (apponto, come nel caso del telelavoro) sia tre le cause della mancata occupazione per le donne con disabilità (cfr. domanda n. 21 - a risposta chiusa con più alternative di risposta)

${ }^{12}$ Questionario con 22 item, 21 a risposta multipla e una a risposta aperta, articolato in tre sezioni: dati sociometrici, settori di impiego in base alle categorie statistiche (ILO, 2018; ISCO-08), settori emergenti in base alle personali esperienze pregresse. 
Fig. 3 - Domanda n. 20 - Fattori che incidono sulla mancata occupazione

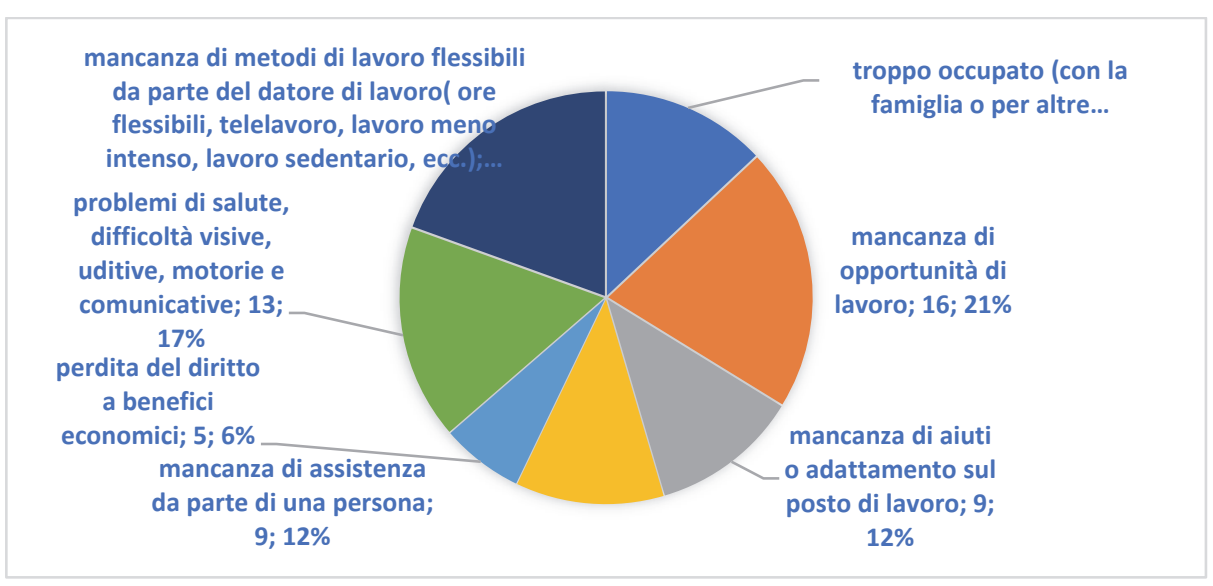

Tali aspetti sono confermati nella domanda a risposta aperta in cui gli operatori sono stati chiamati a descrivere le condizioni che a diverso livello favoriscono l'ingresso nel lavoro da parte delle donne con disabilità (cfr. domanda n. 22 - risposta aperta, analizzata tramite metodo QDA, Charmaz, 2005):

Tabella 6 - Categorie core emergenti, domanda a risposta aperta n. 22

\begin{tabular}{|c|c|c|}
\hline Competenze necessarie & Adattamenti delle aziende & Macro-condizioni \\
\hline $\begin{array}{l}\text { 1. autonomia e flessibilità sul la- } \\
\text { voro (es. smart working/telela- } \\
\text { voro) } \\
\text { 2. abilità interpersonali di comu- } \\
\text { nicazione in lingua straniera e } \\
\text { interazioni con i social media } \\
\text { 3. capacità di proattività legate } \\
\text { alla risoluzione dei problemi } \\
\text { 4. autostima e motivazione } \\
\text { 5. esperienza e competenze } \\
\text { specifiche per una professione } \\
\text { e / o occupazione }\end{array}$ & $\begin{array}{l}\text { 1. formazione specifica all'ini- } \\
\text { zio di un nuovo lavoro } \\
\text { 2. adattamento dell'azienda } \\
\text { alla persona } \\
\text { 3. strategia del lavoro recipro- } \\
\text { camente solidale }\end{array}$ & $\begin{array}{l}\text { 1. istruzione superiore } \\
\text { 2. apprendimento permanente } \\
\text { 3. bassa percentuale di disabi- } \\
\text { lità fisica e intellettuale }\end{array}$ \\
\hline
\end{tabular}

\section{Risultanze e riflessioni su scenari possibili di sviluppo dello smart-work per migliorare l'accessibilità al mondo del lavoro delle donne disabili}

La restituzione dei dati dello studio diagnostico effettuato nel corso della ricerca "Ready woman" ci consente inferenze molteplici su base di evidenze 
circa le potenzialità e le criticità dell'uso dello smart work in condizione di disabilità di tipo sensoriale ed organico, per lo meno in relazione al target considerato nell'indagine.

Nella restituzione procederemo all'esposizione delle risultanze secondo l'ordine sequenziale di utilizzo degli strumenti (par. 3 infra: prima gli esiti dei focus-group alle 106 donne con disabilità, poi quelli del questionario somministrato alle 36 operatrici e infine quelli delle interviste in profondità somministrate a 22 operatrici) attraverso "grandi sintesi" qui di seguito presentate in termini di barriere e di opportunità d'uso dello smart-working.

Cominciamo col dire che l'analisi di contenuto delle rappresentazioni dello smart work (tanto delle donne con disabilità che degli operatori) ha confermato il nesso già ampiamente dimostrato dalla ricerca su disabilità e lavoro (Galarneau \& Radulescu, 2009; Turner, Bohata, Thompson, 2017) circa la relazione lavoro-percezione del valore identitario e sociale della donna con disabilità (Lero, Pletsch, Hilbrecht, 2012; Boffo, Falconi, Zappaterra, 2012; Caldin, Scollo, 2018). Nell'opportunità lavorativa offerta a una persona con disabilità giocano, infatti, un ruolo fondamentale i cosiddetti "impliciti" dell'esperienza lavorativa (Perla, 2010) comprendenti dimensioni "sotto-traccia" della consapevolezza (aspettative, motivazioni, desideri emancipativi) destinati a influenzare l'immagine sociale vissuta e percepita non solo da parte di chi è portatore di una disabilità ma, anche, da parte di chi non è un disabile. L'utilità dei risultati di questo tipo di ricerche risiede, dunque, in un doppio livello di approdi: oltre che nella visibilizzazione del punto di vista delle donne con disabilità (il più delle volte sottostimato), anche nella riconcettualizzazione culturale che accompagna i processi di visibilizzazione di quel punto di vista: la decostruzione del doppio meccanismo discriminativo legato a genere/disabilità sfida, infatti, il concetto di disabilità come onere rafforzando di converso, in chi non è disabile, le rappresentazioni sociali di cittadinanza e di inclusione. L'opportunità del lavoro a una donna portatrice di disabilità contribuisce, dunque, ad aumentare il grado di partecipazione sociale di quest'ultima ma, contestualmente, "spinge" verso l'assimilazione culturale generalizzata del modello sociale di disabilità che ribalta gli stereotipi di improduttività diffusi attraverso l'etichettamento sociale (Tororei, 2009).

\section{Barriere}

Per quanto riguarda la percezione delle barriere connesse all'adozione dello smart-work, sono emersi tre aspetti di natura fondamentalmente psicologica fra loro interagenti: 
a) un fattore individuale che potremmo definire di resistenza "mentale" legato alla scarsa conoscenza dello smart-work unitamente alle dichiarazioni di un fattore di resistenza "pregiudiziale" da parte dei datori di lavoro che, a detta delle donne con disabilità, sarebbero contrari al telelavoro o a qualsiasi forma di smart-work. I loci causali di tali resistenze sono individuati in difficoltà (temute e/o vissute): lo smart-work creerebbe isolamento, richiederebbe il possesso di competenze perlopiù non possedute $\mathrm{e}$, soprattutto, se ne sa poco. La genericità delle risposte date al riguardo fa inferire una scarsissima conoscenza da parte delle donne con disabilità della differenza fra telelavoro e smart-work ${ }^{13}$ (si portano argomenti basati su credenze e opinioni: lo smart-work isola, non è un "vero" lavoro ecc.).

b) un fattore di resistenza, anche questo di natura psicologica, legato alla disistima di sé che impedirebbe in generale l'accesso all'impiego lavorativo, sia in presenza che in modalità "agile";

c) infine un fattore di diffidenza spiegato alla luce di timori di natura contrattuale e, di conseguenza, retributiva.

Gli operatori in effetti confermano l'esistenza di gravi problemi di comunicazione tra datori di lavoro, agenzie di collocamento e donne con disabilità. Molte barriere sono nel campo dell'istruzione e della formazione professionale e queste impediscono la qualificazione necessaria a ricoprire posizioni che richiedano competenze di tipo informatico-tecnologico, indispensabili per l'accesso a telelavoro e smart-work. Gli operatori denunciano poi la loro personale "difficoltà" nel gestire la dinamica relazionale con le lavoratrici disabili che enfatizzino costantemente la propria disabilità oppure cercano di "nasconderla" in tutti i modi possibili. Sfortunatamente, in generale, l'atteggiamento dei datori di lavoro verso le donne con disabilità, e in particolare verso le persone con problemi psicologici, è a priori negativo. Pertanto, sono pochi quelli che scelgono di dichiarare apertamente le complessità legate alla disabilità in un colloquio di lavoro: le interessate temono che la loro domanda non sarà considerata equivalente $\mathrm{o}$, all'inverso, che sarà considerata come un tentativo di ottenere un trattamento preferenziale.

Una barriera del tutto specifica, e fondata questa volta su dati di realtà, è quella che riguarda il mancato possesso di competenze informatiche che 'sono

\footnotetext{
${ }^{13}$ Esistono differenze fra l'uno e l'altro. Per telelavoro si intende un lavoro che si svolge a distanza rispetto alla sede centrale e il datore è legittimato ad effettuare verifiche ispettive nel luogo deputato allo svolgimento delle azioni. Nello smart-work, invece, non è obbligatorio legarsi a un luogo fisico fisso in cui lavorare. Anche la propria abitazione va bene o qualsiasi luogo ove si possa portare un computer o uno smartphone e sia presente una connessione Wi-Fi. Nello smart-work anche l'orario è autodeterminato: l'importante è raggiungere l'obiettivo prefissato e il monte ore è gestito dal lavoratore che può, in tal modo, evitare $i$ costi degli spostamenti e tenere in equilibrio più facilmente vita familiare e vita lavorativa.
} 
uno dei maggiori problemi per le persone con disabilità' in quanto, se da un lato le tecnologie sono ritenute 'uno strumento di lavoro utile per le donne con disabilità', dall'altro lato il loro utilizzo limiterebbe la partecipazione diretta ai contesti lavorativi. Ritorna qui la preoccupazione delle donne di una presunta "inibizione" della modalità smart-work del processo inclusivo: anche per questo la formazione al saper usare le tecnologie dell'informazione è ritenuto un requisito necessario per il miglioramento della qualità della vita tout court, e non solo per il miglioramento delle potenzialità di accesso. Come è possibile evincere da questa breve sintesi, le resistenze alla sperimentazione dello smartwork da parte delle donne con disabilità sono in grande maggioranza frutto di rappresentazioni soggettive, poco negoziate nella dialettica con gli operatori, anche perché poco presenti alla "consapevolezza" delle stesse donne.

\section{Opportunità}

Tali rappresentazioni sembrano stridere con quelle degli operatori i quali vedono, invece, nella modalità lavorativa "smart" una concreta opportunità da valorizzare ai fini dell'accessibilità nel mercato del lavoro da parte delle donne con disabilità.

Tabella 7 - Risultati

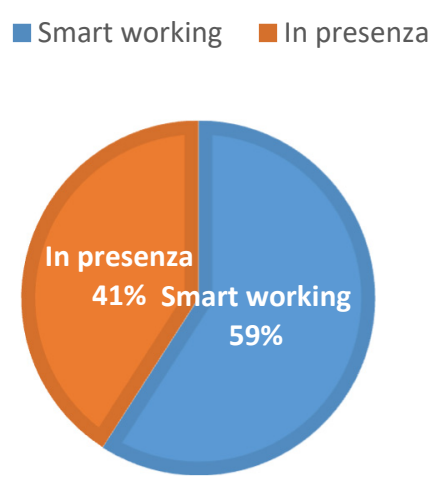

Come è possibile osservare in tab. 7, il 59\% degli operatori intervistati ha dichiarato che lo smart work è uno "strumento" che facilita le possibilità di accesso al lavoro delle donne con disabilità, specie nelle piccole imprese e negli ambiti della contabilità, del web design, della grafica, delle traduzioni. Ovviamente tale opportunità si lega a doppio filo a quella dell'organizzazione di una formazione rispondente al bisogno dichiarato dalle donne con disabilità e confermato anche dagli operatori intervistati. È, dunque, su una formazione alle 
competenze digitali e informatiche che occorrerà posare l'attenzione nel prosieguo della ricerca. Il focus sarà, dunque, su una mediazione digitale personalizzata per migliorare agency e qualità della vita delle persone con disabilità (Perla, 2013, Perla Agrati, 2018; Giaconi, 2015) in vista dell'accesso alle possibilità offerte dallo smart-work e l'uso di strumenti specifici ma, anche, finalizzata a rispondere ai bisogni di cui ogni cittadino della società dell'informazione e comunicazione è portatore: bisogno di essere informato, bisogno di interagire, bisogno di esprimersi, bisogno di protezione, bisogno di gestire situazioni complesse connesse agli strumenti tecnologici e agli ambienti digitali. Anche per questo, la seconda fase della ricerca articolerà le azioni per il disegno di un curricolo inclusivo di formazione anche alle competenze di cittadinanza digitale, trasversali e soft nonché di tipo attitudinale per la cui validazione si attenderà la chiusura del progetto.

\section{Bibliografia}

Angeloni S. (2010). L'aziendabilità. Il valore delle risorse disabili per l'azienda e il valore dell'azienda per le risorse disabili. Milano: FrancoAngeli.

Atkinson A. B. (1998). Social Exclusion, Poverty and Unemployment. In A. B. Atkinson, \& J. Hills (Eds.). Exclusion, Employment and Opportunity. Centre for Analysis of Social Exclusion (CASE). London UK: London School of Economics and Political Science, pp 1-20.

Atkinson R. (1998). The Life Story Interview. London: Sage.

Bazeley P. \& Jackson K. (2013). Qualitative Data Analysis with NVivo. London: Sage.

Boffo V., Falconi S., Zappaterra T. (a cura di) (2012). Per una formazione al lavoro. Le sfide della disabilità adulta. Firenze: Firenze University Press.

Brinkmann S., \& Kvale S. (2015). Interviews: Learning the craft of qualitative research interviewing (Vol. 3). Thousand Oaks, CA: Sage.

Bryman A. (2012). Social research methods (4th ed.). Oxford: Oxford University Press.

Cairo M., Mariani V., Zoni, Confalonieri R. (2010). Disabilità ed età adulta. Qualità della vita e progettualità pedagogica. Milano: Vita e Pensiero.

Cairo M., Marrone M. (2017). Qualità della vita, narrazione e disabilità. Esperienze e proposte. Milano: Vita e Pensiero.

Caldin R., Scollo S. (2018). Inclusione lavorativa, disabilità e identità. Riflessioni e rappresentazioni. Studium Educationis, XIX(3): 49-59.

Calvani A. (2017). Manuale di tecnologia dell'educazione. Pisa: Edizioni ETS.

Cameron R. (2015). The emerging use of mixed methods in educational research. In M. Baguley, Y.S. Findlay \& M.C. Karby (Eds). Meanings and motivation in education research, Chapter 8. New York: Routledge.

Charmaz K. (2005). Grounded Theory in the $21^{\circ}$ Century. In N. K. Denzin \& Y. S. Lincoln. (Eds). Handbook of Qualitative Research, Terza Ed, Thousand Oaks: Sage. 
CISCO (2011). Work-Life Innovation Smart Work - A Paradigm Shift Transforming How, Where, and When Work Gets Done, http://www.cisco.com/web/about/ac79/docs/ps/Work-Life_Innovation Smart Work.pdf.

Colazzo S. (2010). I problemi dell'accesso. In Paparella N., Rocca N. (ed.) (2010). Disabili mentali. Cittadini a pieno titolo. Lecce: Edizioni di solidarietà.

Corbetta P. (2003). La ricerca sociale: metodologia e tecniche. Bologna: il Mulino.

Cottini L., Fedeli D. \& Zorzi S. (2016). Qualità della vita nella disabilità adulta. Percorsi, servizi e strumenti psicoeducativi. Trento: Erickson.

Creswell J. W. (2007). Second Edition: Qualitative inquiry \& research design. Choosing Among Five Approaches. London: Sage.

Creswell J. W., Plano Clark V. L., Guttman M. \& Hanson W. (2003). Advanced mixed methods research designs. In A. Tashakkori, C. Teddlie \& C.B. Teddlie. (Eds.). Handbook of mixed methods in social \& behavioral research, pp. 209-240. Thousand Oaks, CA: Sage.

D’Alonzo L. (2018). Pedagogia Speciale per l'inclusione. Brescia: Editrice Morcelliana Scholé.

European Commission (2017). Progress Report on the implementation of the European Disability Strategy (2010-2020). EU Bruxelles.

Eurostat (2019). Statistiche dell'occupazione. https://ec.europa.eu/eurostat/statisticsexplained/index.php?title=Employment_statistics/it.

Fiore P. (2018). Disabilità e luoghi di lavoro. In AA.VV. Il piano di eliminazione delle barriere architettoniche: Un approccio integrato alla progettazione. Milano: FrancoAngeli.

Frisina A. (2010). Focus group. Una guida pratica. Bologna: il Mulino.

Galarneau D. \& Radulescu M. (2009). Employment among the disabled. Perspectives on Labour and Income, 10(5): 5-15.

Giaconi C. (2015). Qualità della vita e adulti con disabilità. Percorsi di ricerca e prospettive inclusive. Milano: FrancoAngeli.

Gobo G. (2004). Generalizzare da un solo caso? Lineamenti di una teoria idiografica dei campioni. Rassegna italiana di sociologia, 1: 1-27. DOI: 10.1423/13200.

Gottlieb A., Myhill W. N., \& Blanck P. (2010). Employment of People with Disabilities. In: J.H. Stone, M. Blouin (Eds). International Encyclopedia of Rehabilitation. Buffalo: Center for International Rehabilitation Research Information and Exchange (CIRRIE).

Horn-Haacke L. (2002). Using SWOT for Project Team Planning Sessions. GFA, Website: www.gfa-group.de/publications/webdownloads/1054/Swot.pdf.

Istat (2019). Conoscere il mondo della disabilità. Persone, relazioni e istituzioni. Roma: Istituto nazionale di statistica.

Istat (2019). Occupati e disoccupati. Dati provvisori. https://www.istat.it/it/files//2019/11/CS_Occupati-e-disoccupati_OTTOBRE_2019.pdf.

Jedlowski P. (2000). Storie comuni. La narrazione nella vita quotidiana. Milano: Mondadori. 
Kadouche R., Abdulrazak B., Giroux S., Mokhtari M. (2009). Disability centered approach in smart space management. International Journal of Smart Home, 3(2), April.

Leech N. \& Onwuegbuzie A. J. (2009). A typology of mixed methods research designs, Quality and Quantity, 43(2): 265-275.

Lero D., Pletsch C., Hilbrecht M. (2012). Introduction to the Special Issue on Disability and Work: Toward Re-Conceptualizing the 'Burden' of Disability. Disability Studies Quarterly, 32(3).

Lewins A. \& Silver C. (2007). Using software in qualitative research: A step by step guide. London: Sage.

Mann W.C. (2005) (Ed.). Smart technology for aging, disability, and independence: The state of the science. Hoboken, NJ: John Wiley \& Sons.

Marciano C. (2012). Unpacking a smart city model. International Journal of Interdisciplinary Social Sciences - section Communication Studies, Chicago, USA: University of Chicago Illinois.

Mattana V. (2016), Il disability managment in Italia: una rassegna degli studi basati su evidenza Impresa Progetto. Electronic Journal of Management, 1.

Ministero degli Affari Esteri. XVI Legislatura. (2007). La Convenzione delle Nazioni Unite sui diritti delle persone con disabilità. www.senato.it/bgt/pdf/s1279cnv2.pdf.

Ministero del Lavoro e delle Politiche Sociali (2014). Smart Ecnomy. Green Jobs - Formazione e Orientamento. https://www.cliclavoro.gov.it/Progetti/Green_Jobs/Documents/Smart_City/3_Smart\%20Economy.pdf.

Mitra S. (2006). The Capability Approach and disability. In Journal of Disability Policy Studies, 16(4): 236-247.

Pacifico M. \& Coppola L. (2010). NVivo: una risorsa metodologica. Procedure per l'analisi dei dati qualitativi. Milano: FrancoAngeli.

Paparella N. (2010). Il progetto BES. In Paparella N., Rocca N. (ed.) (2010). Disabili mentali. Cittadini a pieno titolo. Lecce: Edizioni di solidarietà.

Perla L. (2010). Didattica dell'implicito. Brescia: La Scuola.

Perla L. (2013). Per una didattica dell'inclusione. Prove di formalizzazione. BresciaLecce: Pensa Multimedia.

Perla L., Agrati L. (2018). L'agentività dell'insegnante inclusivo. Uno studio esplorativo sul Coordinatore per l'inclusione. In Sibilio M., Aiello P. (Eds.). Lo sviluppo professionale dei docenti. Ragionare di agentività per una scuola inclusiva, pp. 239-258. Napoli: EdiSES.

Perla L. (2020). Didattica e pratiche dell'active learning. In Crescenza G., Volpicella A. (2020), Apprendere a insegnare. Competenze e sensibilità della professione docente, Roma: Edizioni Conoscenza, pp. 264-274.

Ravicchio F., Repetto M., \& Trentin G. (2015). Formazione in rete, teleworking e inclusione Lavorativa. Milano: FrancoAngeli.

Ravicchio V., Repetto M., \& Trentin G. (2015). Formazione formatori sullo smart working per disabili: come valutarne le ricadute. TD Tecnologie Didattiche, 23(2): 102-111.

Robertson P. (2018). Building capabilities in disabled job seekers: A qualitative study of the Remploy Work Choices programme in Scotland. Social Work and Society, 16(1). 
Sallis A., Birkin R. (2014). Experiences of work and sickness absence in employees with depression: An interpretative phenomenological analysis. Journal of Occupational Rehabilitation, 24: 469-483.

Samant Raja D. (2016). Bridging the disability divide through digital technologies. Background Paper for the 2016 World Development Report: Digital Dividends, World Bank Group.

Sellevoll K. T. (2016). Disability and work Barriers to employment. A qualitative study on disability and experiences entering the labor market. Master Thesis, Hedmark.

Sen A. (1999). Development as freedom. Oxford: Oxford University Press.

Strauss A., \& Corbin J. (1990). Basics of Qualitative Research: Grounded Theory Procedures and Techniques. Newbury Park: Sage.

Tarozzi M. (2016). Che cos'è la grounded Theory. Roma: Carocci.

Tororei S.K. (2009). The right to work: A strategy for addressing the invisibility of persons with disability. Disability Studies Quarterly, 29(4).

Trani J.F., Bakhshi P., Bellanca N., Biggeri M., Marchetta F. (2011). Disabilities through the Capability Approach lens: Implications for public policies. Handicap au travers de l'approche par les capabilités: quelles implications pour les politiques publiques? Alter: European journal of disability research, 5(3): 143-157.

Turner D.M., Bohata K., Thompson S. (2017). Work and Representation. New Perspectives, 37(4)

Vedeler J. S. (2014). Disabled persons'job interviews experiences: stories of discrimination, uncertainty and recognition. Disability \& Society, 29(4): 597-610. DOI: 10.1080/09687599.2013.831748.

Volpicella A.M. (2017). La pedagogia dell'inclusione. In Volpicella A.M., Crescenza G. (a cura di). Una bussola per la scuola. Nuove strategie pedagogiche e didattiche per gli studenti di oggi. Roma: Edizioni Conoscenza.

Vornholt K., Villotti P. et al. (2018). Disability and employment - overview and highlights. European Journal of Work and Organizational Psychology, 27(1): 40-55. DOI: $10.1080 / 1359432 X .2017 .1387536$.

\section{Sitografia}

European Disability Forum. Fonte: www.edf-feph.org.

QSR International: www.qsrinternational.com. 